\title{
Enxertia interespecífica de jabuticabeira: influência do tipo de garfo
}

\author{
Interspecific grafting of jabuticabeira: influence the type of stem cutting
}

\author{
Gustavo Malagi ${ }^{\mathrm{I}}$ Idemir Citadin $^{\mathrm{I} *}$ Silvia Scariotto ${ }^{\mathrm{III}}$ Américo Wagner Júnior ${ }^{\mathrm{II}}$ \\ Marcos Robson Sachet ${ }^{\mathrm{III}}$
} \begin{abstract}
Este trabalho objetivou avaliar o pegamento e
desenvolvimento dos enxertos de duas espécies de Jabuticaba
(Plinia spp.), testando diferentes tipos de garfos. Utilizou-se o
delineamento inteiramente casualizado, com dez repetições,
no esquema fatorial $2 x 3$ (espécie x tipo de garfo), com a unidade
experimental composta por uma planta. Utilizaram-se as
espécies Plinia trunciflora e Plinia cauliflora enxertadas sobre
P. cauliflora por garfagem de topo em fenda cheia, utilizando-
se garfos herbáceos (diâmetro menor de 3mm), semi-lenhosos
de baixo calibre (diâmetro de 3-5mm) e semi-lenhosos de
médio calibre (diâmetro de 5-7mm). A espécie P. trunciflora
proporcionou um número de brotações superior e um
comprimento de brotação $44 \%$ inferior à espécie P. cauliflora.
Para percentual de brotação, não se observou diferença
significativa entre as espécies testadas. Os garfos semi-lenhosos
de baixo calibre favoreceram o aumento significativo do
pegamento e do número de brotação em comparação aos
garfos herbáceos, não diferindo significativamente, no entanto,
dos garfos semi-lenhosos. RESUMO
\end{abstract}

Palavras-chave: diâmetro do garfo,garfo herbáceo, garfo semi-lenhoso.

\section{ABSTRACT}

This research aimed to evaluate craft success and development of two grafted species of Jabuticaba (Plinia spp.) using different methods of grafting. A Complete randomized experimental design was used with ten replicates, under a factorial scheme of $2 \times 3$ (species $x$ diameter of stem cutting), and the experimental unit was composed by one plant. Plinia trunciflora and Plinia cauliflora were the scion species grafted on $\boldsymbol{P}$. cauliflora by cleft grafting, using herbaceous cuttings (diameters smaller than $3 \mathrm{~mm}$ ), semi-woody cuttings of low caliber (diameter 3-5mm), and semi-woody cuttings of medium caliber (diameter $5-7 \mathrm{~mm}$ ). P. trunciflora presented the higher number of buddings but the length of buddings were 44\% lower than $\boldsymbol{P}$. cauliflora. Budding percentage was not significantly different among studied species. The semi-wood cuttings of low caliber increased significantly the number of buddings and graft success when compared to herbaceous cuttings, not presenting significant difference when compared to semi-woody cuttings of medium caliber herbaceous cuttings.

Key words: diameter of stem cutting, herbaceous cutting, semiwoody cutting.

O Brasil apresenta grande diversidade de espécies frutíferas, sendo, no entanto, ainda pouco conhecidas, além de serem exploradas quase que exclusivamente pelo extrativismo. Considerando-se a variação climática do país frente a sua dimensão continental, as espécies frutíferas brasileiras estão distribuídas em todos os biomas e, portanto, a importância de cada espécie é relativa à região de exploração. Dentre as espécies frutíferas, destaca-se a jabuticabeira (Plinia spp.) Berg., que pode ser encontrada nas regiões Nordeste, Centro Sul, Sudeste e Centro-Oeste, distribuída nos biomas da Caatinga, Cerrado, Mata Atlântica e Pantanal (RASEIRA et al., 2004). Porém, mesmo conhecendo o potencial comercial dos frutos, a cultura não desperta atenção para o cultivo em grande escala, pois a planta demora a entrar

'Fruticultura de Clima Temperado, Programa de Pós-graduação em Agronomia, Faculdade de Agronomia 'Eliseu Maciel' (FAEM), Universidade Federal de Pelotas (UFPel), Campus Universitário Capão do Leão, Pelotas, RS, Brasil.

IIPrograma de Pós-graduação em Agronomia, Universidade Tecnológica Federal do Paraná (UTFPR), Via do conhecimento, km 01, 85503-390, Pato Branco, PR, Brasil. E-mail: idemir@utfpr.edu.br. *Autor para correspondência.

IIILaboratório de Fruticultura, UTFPR, Pato Branco, PR, Brasil. 
em produção (DANNER et al., 2006), podendo o período variar entre oito e quinze anos após o plantio, quando multiplicadas por semente, pois as plantas não conseguem superar o período juvenil tão rapidamente (ANDRADE \& MARTINS, 2003).

Nesse sentido, a possibilidade de usufruir das vantagens da jabuticaba parece estar restrita ainda a sua propagação. A propagação pelo método da estaquia é dificultada em razão do difícil enraizamento (SASSO et al., 2010b). Assim, evitando-se o insucesso pela técnica da estaquia e para que o período juvenil das plantas seja eliminado, pode-se optar pelo uso da enxertia ou da alporquia (SASSO et al., 2010a), que favorece ainda o início da produção em um menor espaço de tempo. A técnica de enxertia por garfagem em fenda cheia pode variar de acordo com a espécie utilizada e a época da enxertia. SILVA et al. (2008), testando as espécies $\boldsymbol{P}$. jaboticaba (Vell) Berg. e $\boldsymbol{P}$. cauliflora (DC) Berg., enxertadas sobre porta-enxerto da mesma espécie, por garfagem de topo em fenda cheia, obtiveram pegamento de 93,75\% para $\boldsymbol{P}$. jaboticaba e 68,75\% para $\boldsymbol{P}$. cauliflora (DC) Berg.

Mesmo que seja notável a eficiência da enxertia em jabuticaba, são necessários ainda estudos complementares que permitam conjuntamente recomendar a técnica da enxertia como ferramenta no desenvolvimento do cultivo comercial da jabuticabeira. Assim, objetivou-se com o presente trabalho avaliar o efeito do tipo e diâmetro de garfo na enxertia de duas cultivares de jabuticabeiras pelo método de garfagem de topo em fenda cheia.

O experimento foi conduzido na casa de vegetação da Universidade Tecnológica Federal do Paraná, UTFPR, Campus Pato Branco, em 2009. Foi testada a compatibilidade de enxertia de duas espécies de jabuticabeira (P. cauliflora (DC) Berg., P. trunciflora (O. Berg.) Kausel) sobre o porta-enxerto $\boldsymbol{P}$. cauliflora (DC) Berg., espécie de ocorrência típica na região sudoeste do estado do Paraná. Os porta-enxertos foram originários de sementes semeadas em setembro de 2006, em sacos plásticos com volume de $2000 \mathrm{~cm}^{3}$, contendo LATOSSOLO VERMELHO DISTROFÉRICO e substrato Plantmax ${ }^{\odot}$ (mudas florestais) na proporção de 1:1 (v/v). Plantas matrizes das espécies $\boldsymbol{P}$. cauliflora e $\boldsymbol{P}$. trunciflora, já adultas (35-40 anos), fora do período reprodutivo, localizadas no município de Itapejara D’Oeste, PR, foram utilizadas para a coleta de ramos e formação dos garfos.

Utilizou-se o delineamento experimental inteiramente casualizado, em esquema fatorial $2 \mathrm{x} 3$ (espécies $\mathrm{x}$ tipos de garfos) com 10 repetições, totalizando 60 plantas. Para cada espécie, foram preparados três diferentes tipos de garfos: herbáceos (<3mm de diâmetro), semi-lenhosos de baixo calibre (3$5 \mathrm{~mm}$ de diâmetro) (1) e semi-lenhosos de médio calibre (5-7mm de diâmetro) (2) com $10 \mathrm{~cm}$ de comprimento.

Os garfos foram enxertados em ramos com diâmetros aproximados, pela técnica da garfagem de topo em fenda cheia, $15 \mathrm{~cm}$ acima do nível do solo. A extremidade do garfo e a região da enxertia foram cobertas com fita de enxertia, tipo Buddy-tape. Foram mantidos alguns ramos (ramos pulmões) nos portaenxertos até que os brotos dos garfos atingissem aproximadamente $10 \mathrm{~cm}$ de altura, para facilitar a atividade fotossintética da planta e favorecer o pegamento do enxerto, sendo retirados em seguida.

A enxertia foi realizada no mês de maio de 2009, sendo os enxertos mantidos em casa de vegetação sob temperatura controlada (mínima: $15^{\circ} \mathrm{C}$; máxima: $28^{\circ} \mathrm{C}$ ), abrigados do excesso de luz e suplementados com irrigação por aspersão. Cento e vinte dias após a enxertia, verificaram-se o percentual de brotação, número e comprimento das brotações dos enxertos.

Os dados obtidos foram submetidos à análise de normalidade, homocedasticidade e independência dos erros. Os dados relativos às variáveis 'número' e 'comprimento das brotações’ não apresentaram normalidade e foram transformados por $\sqrt{(x+0,5)}$. Em seguida, esses dados, juntamente com aqueles da variável 'percentual de brotação', foram submetidos à ANOVA, seguido de comparação múltipla de médias pelo teste Tukey $(\mathrm{p} \leq 0,05)$.

A brotação dos enxertos ocorreu simultaneamente no $85^{\circ}$ dia após a enxertia em todos os tratamentos, sem que diferenças significativas fossem observadas. Considerando-se o percentual de brotação, número e comprimento das brotações, não foi possível identificar interação significativa entre as espécies x diâmetro dos garfos, pela análise de variância.

Em relação ao percentual de brotação, $\boldsymbol{P}$. cauliflora e $\boldsymbol{P}$. trunciflora apresentaram percentuais de brotação de 53,3 e 70\%, respectivamente. No entanto, mesmo que para esta variável tenha ocorrido variação entre as duas espécies, essa diferença não foi considerada significativa pelo teste F (Tabela 1). É possível que esta amplitude, observada entre os percentuais de brotação de ambas as espécies, tenha ocorrido em razão de contrastes morfológicos entre os porta-enxertos e as plantas utilizadas como garfos, uma vez que se desenvolveram em condições edafoclimáticas distintas, antes da realização da enxertia, muito embora as plantas matrizes que cederam os garfos, para ambas as espécies, tenham se desenvolvido no mesmo local. 
Enxertia interespecífica de jabuticabeira: influência do tipo de garfo.

Tabela 1 - Percentual de brotação, número de brotações e comprimento das brotações para as espécies $\boldsymbol{P}$. cauliflora (DC) Berg. e $\boldsymbol{P}$. trunciflora (O. Berg.) Kausel enxertadas sobre P. cauliflora (DC) Berg., utilizando garfos com diâmetro de secção <3mm (Herbáceos), de 3 a 5mm (Semi-lenhoso 1) e de 5 a 7mm (Semi-lenhoso 2). UTFPR, campus Pato Branco, 2011.

\begin{tabular}{lccc}
\hline Espécie & Brotação (\%) & Número de brotações & $\begin{array}{c}\text { Comprimento das brotações } \\
(\mathrm{cm})\end{array}$ \\
\hline P. cauliflora (DC) Berg. & $53,3^{\mathrm{NS}}$ & $2,06 \mathrm{~b} * *$ & $11,1 \mathrm{a}$ ** \\
P. trunciflora (O. Berg.) Kausel & 70,0 & $4,20 \mathrm{a}$ & $6,25 \mathrm{~b}$ \\
Média & 61,6 & 3,13 & 8,69 \\
Herbáceo & $45,0 \mathrm{~b} *$ & $2,7 \mathrm{~b} *$ & $7,41^{\mathrm{NS}}$ \\
Semi-lenhoso 1 & $80,0 \mathrm{a}$ & $3,4 \mathrm{a}$ & 10,0 \\
Semi-lenhoso 2 & $60,0 \mathrm{ab}$ & $3,3 \mathrm{ab}$ & 8,61 \\
Média & 61,6 & 3,13 & 8,69 \\
CV(\%) & 67,8 & 8,4 & 22,4 \\
\hline
\end{tabular}

SASSO et al. (2010 a), utilizando três espécies de jabuticabeira, $\boldsymbol{P}$. cauliflora, $\boldsymbol{P}$. trunciflora e $\boldsymbol{P}$. jaboticaba, enxertadas sobre $\boldsymbol{P}$. cauliflora, nos meses de maio e agosto, verificaram que, mesmo não existindo diferença significativa entre as épocas, o mês de maio $(63,9 \%)$ proporcionou as maiores médias de brotação em comparação a agosto (44,5\%). Das três espécies testadas, apenas $\boldsymbol{P}$. jaboticaba apresentou diferença significativa, mostrando apenas $15,6 \%$ de brotação em agosto, fato que, segundo os autores, ocorreu em virtude da espécie estar no período reprodutivo na época da coleta dos garfos, o que pode ter contribuído para o consumo de carboidratos de reserva e impedido a ótima brotação.

A espécie $\boldsymbol{P}$. trunciflora apresentou um número de brotações significativo, cerca de duas vezes $(4,20)$ mais que $\boldsymbol{P}$. cauliflora. É provável que o comprimento das brotações tenha sido influenciado pelo número de brotações por planta, ou seja, o maior número de brotações observado para $\boldsymbol{P}$. trunciflora aumentou a competição por carboidratos e/ou fotoassimilados, reduzindo o comprimento das brotações. O comprimento das brotações foi $44 \%$ menor para a espécie $\boldsymbol{P}$. trunciflora em relação à espécie $\boldsymbol{P}$. cauliflora, ficando explícito que a relação entre número de brotações por planta e comprimento é inversamente proporcional (Tabela 1). Esse resultado sugere que, após a emissão de brotos pelo enxerto, seja realizado o desbrote, o que pode contribuir para o desenvolvimento mais vigoroso dos ramos remanescentes.

Quanto ao garfo utilizado na enxertia, os semi-lenhosos (1) apresentaram uma brotação média de $80 \%$, com média de 3,4 brotações por planta, sendo esses valores significativamente superiores aos observados para garfos herbáceos, porém significativamente iguais aos resultados obtidos pelos garfos semi-lenhosos (2). Para comprimento das brotações, não se observaram diferenças significativas entre os tipos de garfos (Tabela 1). FRANZON et al. (2010), testando a enxertia de sete genótipos de Eugenia uniflora L. por garfagem em fenda cheia, sobre porta enxertos herbáceos da mesma espécie, com diâmetro de 2,5mm na região da enxertia, verificaram pegamento máximo de 87,5\% e mínimo de $40 \%$ entre os genótipos.

As observações deste estudo em relação ao tipo de garfo utilizado na enxertia demonstram que os garfos herbáceos apresentam maior dificuldade de pegamento, possivelmente pela maior possibilidade de perda de água e pela maior facilidade de oxidação dos tecidos. Ainda, é possível que o potencial de soldadura dos enxertos tenha sido limitado pela maior fragilidade do conjunto celular do floema, do câmbio e do xilema, que pode ter sofrido danos durante a preparação dos garfos e/ou durante o processo de enxertia. Quanto aos garfos semi-lenhosos de maior diâmetro, é possível que seu estado de maior lignificação em relação aos demais tenha contribuído para a redução do pegamento dos enxertos.

À medida que as plantas lenhosas jovens se desenvolvem, como é o caso da Jabuticabeira, a lignificação do caule aumenta, de modo que o sucesso da técnica de enxertia é limitado pela dificuldade de cicatrização e união cambial do enxerto e porta-enxerto (FRANZON et al., 2008). Em espécies da família Myrtaceae, é comum a oxidação de compostos fenólicos durante o procedimento de enxertia, o que contribui para a menor formação de calos e cicatrização no local da enxertia (FACHINELLO et al., 2005). Em razão disso, FRANZON et al. (2008) recomenda a enxertia de pitangueira em porta enxertos da própria espécie com diâmetro entre 2 e $4 \mathrm{~mm}$ na região de enxertia.

Os resultados obtidos neste trabalho contribuem para o aperfeiçoamento da técnica de enxertia na produção de mudas de jabuticabeira. Garfo 
do tipo semi-lenhoso de baixo calibre, com diâmetro entre 3 e $5 \mathrm{~mm}$ favorecem um percentual de brotação e um número de brotações superior ao garfo do tipo herbáceo, com diâmetro menor que 3mm, e ao semilenhoso de médio calibre, com diâmetro superior a $5 \mathrm{~mm}$.

\section{REFERÊNCIAS}

ANDRADE, R.A.; MARTINS, A.B.G. Influence of the temperature in germination of seeds of jabuticaba tree. Revista Brasileira de Fruticultura, v.25, n.1, p.197-198, 2003. Disponível em: <http:/ /www.scielo.br/pdf/rbf/v25n1/a56v25n1.pdf>. Acesso em: 04 ago. 2011. doi: 10.1590/S0100-29452003000100056.

DANNER, M.A. et al. Enraizamento de jabuticabeiras ( $\boldsymbol{P}$. trunciflora) por mergulhia aérea. Revista Brasileira de Fruticultura, v.28, n.3, p.530-532, 2006.

FACHINELLO, J.C. et al. Propagação de plantas frutíferas. Brasília: Embrapa Informação Tecnológica, 2005. 221p.

FRANZON, R.C. et al. Propagação vegetativa de genótipos de pitangueira do sul do Brasil por enxertia de garfagem. Revista Brasileira de Fruticultura, v.32, n.1, p.262-267, 2010. Disponível em: <http://www.scielo.br/scielo.php?pid=S010029452010005000003\&script=sci_arttext $>$. Acesso em: 04 ago. 2011. doi: 101590/S0100-29452010005000003.
FRANZON, R.C. et al. Propagação da pitangueira através da enxertia de garfagem. Revista Brasileira de Fruticultura, v.30, n.2, p.488-491, 2008. Disponível em: <http:// w w w. s c i e l o.b r / s c i e l o.p h p ? p i d = S 0100 29452008000200038\&script>. Acesso em: 04 ago. 2011. doi: 10.1590/S0100-29452008000200037.

RASEIRA, M.C.B. et al. Espécies frutíferas nativas do sul do Brasil. Pelotas, RS: Embrapa Clima Temperado, 2004. 122p. (Documentos - 129).

SASSO, S.A.Z. et al. Propagação de jabuticabeira por enxertia e alporquia. Revista Brasileira de Fruticultura, v.32, n.2, p.571-576, 2010a. Disponível em: <http:// w w w. s c i e l o.b r / s c i e l o.ph p ? p i d = S 0100 29452010000200030\&script $=$ sci_arttext $>$. Acesso em: 04 ago. 2011. doi: 10.1590/S0100-29452010005000055.

SASSO, S.A.Z. et al. Propagação de jabuticabeira por estaquia. Revista Brasileira de Fruticultura, v.32, n.2, p.577-583, 2010b. Disponível em: <http://www.scielo.br/ scielo.php?script=sci_arttext\&pid=S010029452010000200031 \&lng=pt\&nrm=iso>. Acesso em: 04 ago. 2011. doi: 10.1590/ S0100-29452010005000054.

SILVA, J.F. et al. Pegamento de variedades de jabuticabas submetidas a dois tipos de enxertia. In: CONGRESSO BRASILEIRO DE FRUTICULTURA, 2008., Vitória, ES. Anais... Vitória, ES: Sociedade Brasileira de Fruticultura, 2008. p.1-1. 